\title{
Testicular failure in a patient with G6PC3 deficiency
}

\author{
Yonatan Yeshayahu',2, Roy Asaf', Gal Dubnov-Raz', Ginette Schiby³, Amos J. Simon ${ }^{1,4}$, Atar Lev ${ }^{1,4}$ and Raz Somech ${ }^{1,4}$
}

\begin{abstract}
BACKGROUND: Glucose-6-phosphatase- $\beta$ (G6PC3) deficiency is characterized by congenital neutropenia and variable developmental disorders, including those of the cardiovascular system and the urogenital system (e.g., cryptorchidism) and a peculiar visibility of subcutaneous veins.
\end{abstract}

METHODS: A patient with clinical findings suggestive of G6PC3 deficiency was investigated. Genetic, hematopathologic, immunologic, and endocrine work-up were performed.

RESULTS: The reported patient had binucleotide deletion mutation in G6PC3 and displayed the full spectrum of clinical manifestations associated with G6PC3 deficiency including neutropenia. The reported patient had normal bone marrow cellularity without increased apoptosis, and his neutrophils displayed normal respiratory burst activity. Endocrine workup revealed low testosterone levels, which did not respond to human chorionic gonadotropin stimulation, extremely elevated luteinizing hormone $(\mathrm{LH})$ and follicle-stimulating hormone (FSH) levels, and undetectable anti-Müllerian hormone, all of which are suggestive of testicular failure or anorchia.

CONCLUSION: Our report extends the knowledge about this syndrome and suggests a role for G6PC3 in testicular differentiation and formation. Urogenital dysmorphism could indeed be unrelated to G6PC3 and secondary to consanguinity. However, given the similar description of urogenital anomalies in previous reports of this syndrome, the dysmorphism in our patient is likely related.

C ongenital neutropenia, defined as the presence of absolute neutrophil count (ANC) of less than 500 cells/ $\mu \mathrm{l}$ blood, overlaps a variety of genetically heterogeneous phenotypic disorders. Neutropenia may be an isolated hematologic finding or associated with involvement of other organs (1). A typical example for the latter is glucose-6-phosphatase- $\beta$ (G6PC3) deficiency that is characterized by congenital neutropenia and variable developmental disorders. The variable phenotype of individuals with G6PC3 deficiency may include cardiovascular malformations, such as atrial septal defect, patent ductus arteriosus, and pulmonary vascular abnormality (which leads to pulmonary hypertension), as well as urogenital disorders (e.g., hydronephrosis, urachal fistulations, and cryptorchidism), inflammatory bowel disease, and a peculiar visibility of subcutaneous veins (2-5). The latter was recently described as the most consistent, nonhematologic feature of G6PC3 deficiency. Recently, a nonsyndromic form of severe congenital neutropenia due to G6PC3 mutations was described, suggesting a correlation between the genotype and the nonhematological phenotype of G6PC3 deficiency (6). G6PC3 is a ubiquitously expressed protein localized at the endoplasmic reticulum (ER), catalyzing the hydrolysis of G6P to glucose (7). The accepted underlying mechanism of neutropenia in G6PC3-deficient patients is premature apoptosis of myeloid progenitor cells associated with signs of increased ER stress (8). Granulocyte colony-stimulating factor (G-CSF) administration improves both human and murine G6PC3-deficient neutrophil counts by modulating apoptosis and energy homeostasis (9). G6Pase- $\beta$-deficient $\left(\mathrm{G}_{\mathrm{C} p c 3^{--}}\right)$ mice also manifest neutrophil dysfunction. Their neutrophils exhibit impaired respiratory burst, chemotaxis, and calcium flux, mimicking the immune phenotype seen in other human neutrophil defects (10). The spectrum of urogenital structural aberrations is wider than previously appreciated with cryptorchidism being the most frequent one (11). Cryptorchidism is an anatomical abnormality, which can be the result of a developmental failure. The reason for the high prevalence of cryptorchidism in G6PC3-deficient patients is still obscure. Here, we have reported the clinical findings of a patient with G6PC3 deficiency and describe his mutation, neutrophil function, and hormonal work-up.

\section{RESULTS}

\section{Clinical Findings}

The patient was a premature, small-for-gestational-age boy born at $35 \mathrm{wk}$ of gestation. He showed impaired sucking and severe neutropenia (ANC: $80 / \mu \mathrm{l}$ blood) at birth. Since birth, he suffered from recurrent respiratory infections, oxygen desaturations, and failed to thrive. Dysmorphic features became evident at 2 mo of age. His parents, who are first-degree cousins of Palestinian descent, already had a boy infant with similar clinical findings, who died at the age of 6 mo due to respiratory failure. Physical examination revealed an ill-looking, pale child, with weight and height below the third percentile for age, a prominent superficial venous pattern upon the trunk and abdomen, mild hepato- and splenomegaly, 2/6 systolic

\footnotetext{
'Pediatric Department, The Edmond and Lily Safra Children's Hospital, Sheba Medical Center, Sackler Faculty of Medicine, Tel Aviv University, Tel Aviv, Israel; ${ }^{2}$ Pediatric Endocrinology Unit, Edmond and Lily Safra Children's Hospital, Sheba Medical Center, Sackler Faculty of Medicine, Tel Aviv University, Tel Aviv, Israel; ${ }^{3}$ Department of Pathology, Sheba Medical Center, Tel Hashomer, Sackler Faculty of Medicine, Tel Aviv University, Tel Aviv, Israel; ${ }^{4}$ Cancer Research Center, Pediatric Immunology Service, Jeffrey Modell Foundation (JMF) Center, Sheba Medical Center, Sackler Faculty of Medicine, Tel Aviv University, Tel Aviv, Israel. Correspondence: Raz Somech (raz.somech@sheba.health.gov.il) 
heart murmur, and slight facial dysmorphism, which prominently included nose and ears. The patient had genital ambiguity, which included micropenis with no erectile tissue palpated and a single urethral orifice at the tip of the glans penis. In addition, an undervirilized bifid scrotum was noted with no scrotal skin rugosity and no gonads palpated. No hirsutism or hyperpigmentation of the skin was noted. Echocardiogram revealed an atrial septal defect (ASD) with left to right shunt, minimal tricuspid regurgitation (TR) with mild pulmonary arterial hypertension, and good systolic function.

\section{Laboratory Findings}

Complete blood count showed mild leukopenia $(5,590 / \mathrm{ml})$ with moderate neutropenia $(690 / \mathrm{ml})$. Bone marrow smears showed normal cellularity with a decreased proportion of neutrophils, shift to the left with increase of immature forms. Bone marrow staining with CaspACE was negative. CaspACE binds to activated caspase and serves as a marker for cell apoptosis (Figure 1). In addition, RNA expression and protein levels of binding immunoglobulin protein (BiP) showed no significant difference between patients' and controls' peripheral blood cells. $\mathrm{BiP}$ is a marker of ER stress-induced apoptosis and is elevated in some congenital neutropenias (12). Cytogenetic and immunophenotypic analyses showed no abnormal findings, and the karyotype was 46XY. Genetic analysis of the G6PC3 gene revealed a homozygous mutation consisting of a homozygous 2 base pair deletion in exon 6 of the gene (c. 765_delAG; p.S255fs), resulting in a frameshift and premature stop codon. Both parents were found to be carriers of the mutation (Figure 2).

\section{Endocrine Testing}

A baseline hormonal profile showed normal levels of insulinlike growth factor I and thyroid function tests (Table 1), which were consistent with a normally functioning pituitary. Low testosterone levels were noted alongside extremely elevated $\mathrm{LH}$ and FSH. Anti-Müllerian hormone (AMH) was undetectable, and a slight increase in dehydroepiandrosterone (DHEA-S) levels and normal androstenedione levels were noted. To determine the adrenal function and androgen synthesis, an adrenocorticotropic hormone (ACTH) stimulation test was performed showing a normal adrenal response with a rise of cortisol from $607 \mathrm{nmol} / \mathrm{l}$ at baseline to $1,297 \mathrm{nmol} / \mathrm{l}$ after $60 \mathrm{~min}$. 17-Hydroxyprogesterone was not elevated at baseline and remained normal $60 \mathrm{~min}$ after ACTH injection (1.3 and $3.1 \mathrm{nmol} / \mathrm{l}$, respectively). Testicular function was assessed using the human chorionic gonadotropin (HCG) stimulation test and measuring testosterone at baseline and at $72 \mathrm{~h}$ after an i.m. HCG injection. Levels of testosterone were low at baseline and after HCG stimulation ( $<1.0$ and $1.9 \mathrm{nmol} / \mathrm{l}$, respectively). On repeated ultrasounds performed at $8 \mathrm{wk}$ and $5 \mathrm{mo}$ of age, no testes or gonadal structures were seen in the scrotum, inguinal canals, or the abdomen. In addition, no Müllerian duct structures were found, including a uterus or fallopian tubes.

\section{Immune Evaluations}

Upon admission, the patient displayed significant neutropenia. An excellent response to G-CSF was achieved, with a rise in the neutrophil count (ranging from 5,010/ml to $9,250 / \mathrm{ml}$ ) following weekly doses of G-CSF (10 $\mu \mathrm{g} / \mathrm{kg})$. Production of both

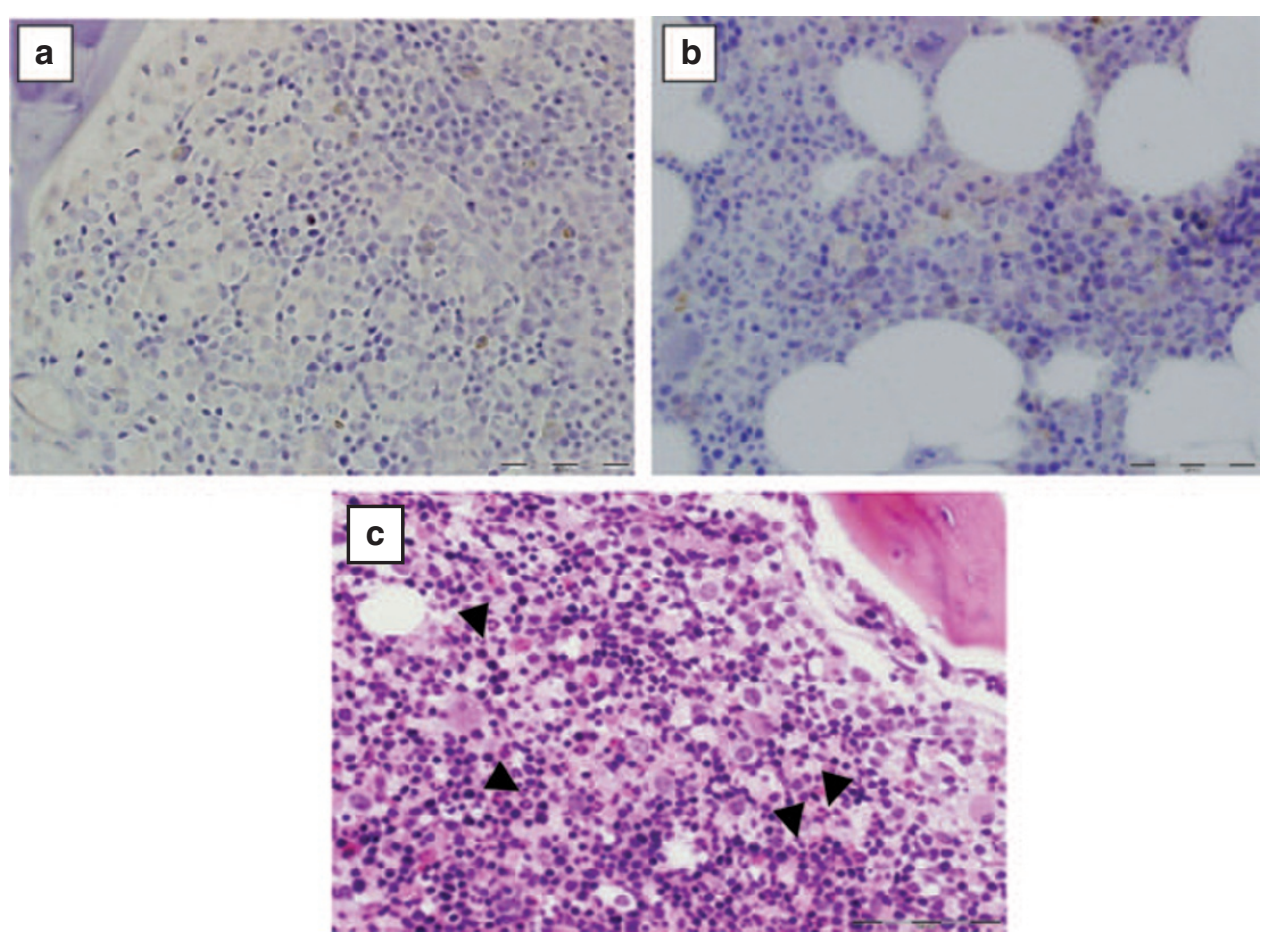

Figure 1. Activated caspase 3 immunostaining. The patient's bone marrow stained with activated caspase 3. No positive cells are identified. (a) Normal bone marrow as negative control, stained with activated caspase 3 with no evidence of apoptotic cells (magnification: $\times 600$ ). (b) H\&E stain (magnification: $\times 600$ ). (c) Section of the patient's bone marrow stained with H\&E showing full myeloid maturation with decreased number of neutrophils (arrowheads indicate neutrophils; magnification: $\times 600)$. H\&E, hematoxylin and eosin. 
a

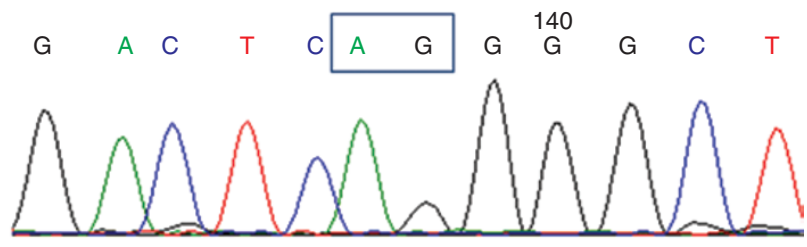

b

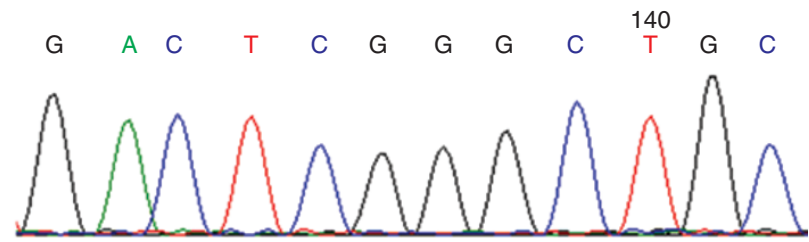

C



Figure 2. Genomic DNA sequence of G6PC3. Exon 7 of the G6PC3 gene was sequenced in healthy control. (a) The patient's DNA sequence. (b) His father's DNA sequence. (c) His mother's DNA sequence. The box indicates the position of the homozygous 2 bp deletion in the patient. The father's sequence shows heterzygocity for the deletion. G6PC3, glucose-6-phosphatase- $\beta$.

hydrogen peroxide and superoxide measured with the DHR$1,2,3$ and the cytochrome $c$ reduction assays, respectively, were normal. Hydrogen peroxide was positive in $99 \%$ of cells with normal activity $>80 \%$ of control. Superoxide was $8.3 \mathrm{nmol} / 10^{6}$ polymorphonuclears $(\mathrm{PMN}) / \mathrm{min}$ (normal range: 1.13-10.2) and $7.5 \mathrm{nmol} / 10^{6} \mathrm{PMN} / \mathrm{min}$ (normal range: 2.03-10.2) in response to formyl-methionyl-leucyl-phenylalanine and phorbol myristate acetate stimulations, respectively. Bactericidal activity, measured as log of decrease of colony-forming unit in response to Staphylococcus aureus infection, was normal (0.46 compared with 0.4 of control).

\section{DISCUSSION}

G6PC3 deficiency causes severe neutropenia and multiple nonhematological abnormalities (11). We reported here about a patient with binucleotide deletion in the G6PC3 gene, which led to a putative frameshift that presumably produced a severely altered and nonfunctional protein product due to a premature stop codon. Similar to the previously reported patient with the same mutation (13), our patient also displayed the full spectrum of clinical manifestations associated with G6PC3 deficiency, including dysmorphic face, congenital neutropenia, prominent superficial venous pattern, cardiac defect, ambiguous genitalia, and cryptorchidism. Most patients with G6PC3 deficiency can be treated successfully with recombinant G-CSF (14), as our patient.

Myeloid maturation arrest of neutrophil precursors at the promyelocyte/myelocyte stage is a common bone marrow feature in G6PC3 deficiency. The molecular pathophysiology of G6PC3 deficiency involves activation of the unfolded protein response, increased ER stress, and increased apoptosis, associated with disturbed glucose homeostasis (2). Our patient displayed normal bone marrow cellularity without maturation block. Furthermore, ER stress was normal, and increased apoptosis was not evident. Similar findings were previously described in other patients with G6PC3 deficiency (15). The reason underlying the variability of bone marrow phenotype and ER stress-induced apoptosis is unclear. Moreover, Cheung et al.(10) have shown not only neutropenia but also neutrophil dysfunction in $\mathrm{G} 6 \mathrm{pc}^{-1-}$ mice. This included reduced neutrophil respiratory burst activity determined by superoxide production after phorbol myristate acetate stimulation. Furthermore, in vivo G-CSF treatment markedly improved respiratory burst and consequently neutrophil function (9). In contrast, we showed that our patient displayed normal respiratory burst activity determined by both superoxide and hydrogen peroxide productions. Yet, neutrophil dysfunction may still be seen in other G6PC3-deficient patients.

Cryptorchidism, micropenis, and ambiguous genitalia, as detected in our patient, are common features in patients with G6PC3 mutations, documented in up to $44 \%$ of patients, and were considered as structural urogenital abnormalities $(2,11,16,17)$. These findings on physical examination are usually the result of either undervirilization in a male or overvirilization in a female. The male karyotype in our patient and lack of internal Müllerian structures suggested that the infant was indeed an undervirilized male. To differentiate between undescended testes and the absence of testicular tissue, hormonal work-up was carried out. The combination of a low testosterone level, unresponsive to HCG stimulation, together with extremely elevated LH and FSH suggests testicular failure or anorchia. $\mathrm{AMH}$, which is normally produced by sertoli cells, was undetectable and supports anorchia rather than cryptorchidism. In the case presented, elevation of both gonadotropins- LH and FSH - suggests an extensive testicular pathology, not limited to Leydig or Sertoli cells. The fact that no gonads were seen on an ultrasound may be consistent with "testicular regression syndrome" ("vanishing testes") and may suggest that G6PC3 plays a role in testicular differentiation and formation. The infant described herein had multiple dysmorphic features, ambiguous genitalia including cryptorchidism. He underwent a full endocrine work-up that demonstrated gonadal failure as an etiology. This is in line with previous studies reporting cryptorchidism and hypogonadism as part of the syndrome. Previous studies of adult patients with G6PC3 deficiency reported males with clinical hypogonadism, which included delayed puberty and gynecomastia. In contrast, females had a slight delay of puberty with normal menarche and menses, which continued during adult life $(11,16)$. No endocrine work-up was reported to have been carried out, and the mechanism of pubertal delay was not explained. To the best of our knowledge, this is the first description of the endocrine properties leading to the genital malformations in infants harboring this mutation in G6PC3. Given the finding of his hypergonadotropic hypogonadism, this child will probably require puberty induction when he reaches adolescence. The fact that male adults reported to have this syndrome suffered from pubertal delay, while female adults had 
Table 1. Baseline and stimulated hormonal levels at the age of 2 mo

\begin{tabular}{|c|c|c|c|}
\hline & Baseline & Stimulated & $\begin{array}{c}\text { Normal } \\
\text { baseline values }\end{array}$ \\
\hline IGF-I (nmol/I) & 7.8 & - & $6-42$ \\
\hline TSH (mlU/l) & 4.27 & - & $0.7-5.9$ \\
\hline Free T4 (pmol/l) & 14.5 & - & $7-16$ \\
\hline $\mathrm{LH}(\mathrm{IU} / \mathrm{I})$ & 41.9 & - & $0.1-3.7$ \\
\hline $\mathrm{FSH}(\mathrm{IU} / \mathrm{I})$ & 121 & - & $0.1-1.9$ \\
\hline Androstendione (nmol/l) & 3.7 & - & $2.0-9.2$ \\
\hline DHEA-S $(\mu \mathrm{mol} / \mathrm{l})$ & 1.7 & - & $0.2-0.6$ \\
\hline $\mathrm{AMH}(\mu \mathrm{g} / \mathrm{l})$ & $<0.16$ & - & $14-466$ \\
\hline HCG stimulation test ${ }^{a}$ & - & - & - \\
\hline Testosterone (nmol/l) & $<1.0$ & 1.9 & $2.6-13.9$ \\
\hline ACTH stimulation test ${ }^{b}$ & - & - & - \\
\hline Morning cortisol (nmol/l) & 607 & 1,297 & $138-690$ \\
\hline 17-OH progesterone (nmol/l) & 1.3 & 3.1 & $<7.5$ \\
\hline
\end{tabular}

Testosterone levels should rise threefold. No testicular response suggests primary hypogonadism.

ACTH, adrenocorticotropic hormone; AMH, anti-Müllerian hormone; DHEA-S,

dehydroepiandrosterone; FSH, follicle-stimulating hormone; HCG, human chorionic gonadotropin; IGF, insulin-like growth factor I; LH, luteinizing hormone; TSH, thyroid stimulating hormone.

aHCG stimulation test: $72 \mathrm{~h}$ after i.m. HCG injection. ${ }^{\mathrm{b}} \mathrm{ACTH}$ stimulation test: $60 \mathrm{~min}$ following intravenous ACTH injection. Normal adrenal response with sufficient rise of cortisol combined with 17-hydroxyprogesterone within the normal range rules out congenital adrenal hyperplasia as an etiology for a virilized female.

normal-onset menses, suggests that the differentiation defect is not gonadal in general but specifically testicular. To note, G6PC3 $3^{-/-}$mice exhibit significant reduction in g6p hydrolytic activity in the testes, although without any known phenotypic consequence (16). Our findings may lead to further assessment of older subjects previously described with this mutation, with regard to hormonal profile, and offer an insight into the role this enzyme plays in sexual differentiation. Our findings need to be interpreted with caution since the patient is the son of consanguineous parents and is prone to dysmorphic features, which may be unrelated to the G6PC3 mutation. However, the finding of no testicular tissue, both anatomically and in hormonal testing, is in line with previous reports of this mutation which included descriptions of cryptorchidism, hypogonadism, and delayed puberty.

In conclusion, we demonstrated here a typical clinical G6PC3-deficient patient yet with atypical immunological findings including normal bone marrow cellularity and ER stress and normal neutrophil respiratory burst activity. Importantly, we provide hormonal work-up showing that cryptorchidism and micropenis, which are typical to the syndrome, at least in our patient, are secondary to gonadal failure rather than just a structural abnormality.

\section{METHODS}

\section{Genetic Work-Up}

Sequencing of the G6PC3 from the patient's genomic DNA was performed as previously described (2). The Institutional Review Board (Sheba Medical Center, Tel Hashomer) approved this study, and written informed consent was obtained from the patient's parents.

\section{Endocrinology Studies}

Adrenal function and androgen synthesis were assessed by performing a $250 \mu \mathrm{g}$ ACTH stimulation test. Testicular function and the presence of testicular tissue were assessed using a HCG stimulation test during which testosterone levels were measured at baseline, and $72 \mathrm{~h}$ following an intramuscular injection of 500 IU of HCG. The HCG stimulation test was done at the age of $5 \mathrm{mo}$.

\section{Neutrophil Studies}

Superoxide production was measured as superoxide dismutase-inhibitable reduction of ferricytochrome $c$. Hydrogen peroxide production was measured with the dihydrorhodamine (DHR)-1,2,3 assay (SigmaAldrich, St Louis, MO). In both assays, neutrophils were stimulated by formyl-methionyl-leucyl-phenylalanine and phorbol myristate acetate (Sigma-Aldrich). All immunological studies were performed at the age of $8-10 \mathrm{wk}$.

\section{Caspase 3 (Active) Immunostaining}

Caspase 3 immunostaining and $\mathrm{BiP}$ expression were performed as previously described (18). Briefly, formalin-fixed tissues were dehydrated, embedded in paraffin, and sectioned at $4 \mu \mathrm{m}$. The slides were warmed up to $60{ }^{\circ} \mathrm{C}$ for $1 \mathrm{~h}$, followed by processing through a fully automated protocol. After the sections were dewaxed and rehydrated, a CC1 Standard Benchmark XT pretreatment for antigen retrieval (Ventana Medical Systems, Tucson, AZ) was selected for caspase 3 (Active). Caspase 3 (Active) antibody, diluted 1:10 (1476-1; Epitomics, Burlingame, CA), was incubated for $1 \mathrm{~h}$ at $37^{\circ} \mathrm{C}$. Detection was performed with an ultraView detection kit (Ventana Medical Systems) and counterstained with hematoxylin (Ventana Medical Systems). The stained sections were viewed and imaged with a light microscope and analyzed by a pathologist. Tissue sampling for pathology was performed at the age of $8-10 \mathrm{wk}$.

\section{BiP Expression}

For $\mathrm{BiP}$ detection, $2-4 \times 10^{6}$ peripheral blood mononucleated cells were harvested, and RNA was extracted using Trizol according to the manufacturer's instructions. cDNA was made from $1 \mu \mathrm{g}$ of total RNA, using the cDNA Archive kit (Applied Biosystems, Grand Island, NY). Real-time quantitative PCR was performed using an ABI 7900 Sequence Detection System (Applied Biosystems). Primers and probes for HSPA5/BiP mRNA, a marker of ER stress, were obtained as assays on demand from Applied Biosystems. The samples were analyzed in triplicates, and fold changes in transcription were calculated using the $\Delta \Delta C_{t}$ comparative method. In all experiments, equal amounts of cDNA were used.

\section{STATEMENT OF FINANCIAL SUPPORT}

This study was supported by The Jeffrey Modell Foundation (JMF), NY, USA; the Legacy Heritage Biomedical Science Partnership Program of the Israel Science Foundation; and the Chief Scientist Office of the Ministry of Health, Israel.

\section{REFERENCES}

1. Klein C. Congenital neutropenia. Hematology Am Soc Hematol Educ Program 2009:344-50.

2. Boztug K, Appaswamy G, Ashikov A, et al. A syndrome with congenital neutropenia and mutations in G6PC3. N Engl J Med 2009;360:32-43.

3. Banka S, Newman WG, Ozgül RK, Dursun A. Mutations in the G6PC3 gene cause Dursun syndrome. Am J Med Genet A 2010;152A:2609-11.

4. Cullinane AR, Vilboux T, O'Brien K, et al. Homozygosity mapping and whole-exome sequencing to detect SLC45A2 and G6PC3 mutations in a single patient with oculocutaneous albinism and neutropenia. J Invest Dermatol 2011;131:2017-25.

5. Bégin P, Patey N, Mueller P, et al. Inflammatory bowel disease and $\mathrm{T}$ cell lymphopenia in G6PC3 deficiency. J Clin Immunol 2013;33:520-5.

6. Banka S, Wynn R, Byers H, Arkwright PD, Newman WG. G6PC3 mutations cause non-syndromic severe congenital neutropenia. Mol Genet Metab 2013;108:138-41.

7. Marcolongo P, Fulceri R, Gamberucci A, Czegle I, Banhegyi G, Benedetti A. Multiple roles of glucose-6-phosphatases in pathophysiology: state of the art and future trends. Biochim Biophys Acta 2013;1830:2608-18. 
8. Boztug K, Klein C. Genetics and pathophysiology of severe congenital neutropenia syndromes unrelated to neutrophil elastase. Hematol Oncol Clin North Am 2013;27:43-60, vii.

9. Jun HS, Lee YM, Song KD, Mansfield BC, Chou JY. G-CSF improves murine G6PC3-deficient neutrophil function by modulating apoptosis and energy homeostasis. Blood 2011;117:3881-92.

10. Cheung YY, Kim SY, Yiu WH, et al. Impaired neutrophil activity and increased susceptibility to bacterial infection in mice lacking glucose6-phosphatase-beta. J Clin Invest 2007;117:784-93.

11. Boztug K, Rosenberg PS, Dorda M, et al. Extended spectrum of human glucose-6-phosphatase catalytic subunit 3 deficiency: novel genotypes and phenotypic variability in severe congenital neutropenia. J Pediatr 2012;160:679-683.e2.

12. Schaffer AA, Klein C. Genetic heterogeneity in severe congenital neutropenia: how many aberrant pathways can kill a neutrophil? Curr Opin Allergy Clin Immunol 2007;7:481-94.
13. Gatti S, Boztug K, Pedini A, et al. A case of syndromic neutropenia and mutation in G6PC3. J Pediatr Hematol Oncol 2011;33:138-40.

14. Estévez OA, Ortega C, Tejero Á, et al. A novel phenotype variant of severe congenital neutropenia caused by G6PC3 deficiency. Pediatr Blood Cancer 2013;60:E29-31.

15. Aytekin C, Germeshausen M, Tuygun N, Dogu F, Ikinciogullari A. A novel G6PC3 gene mutation in a patient with severe congenital neutropenia. J Pediatr Hematol Oncol 2013;35:e81-3.

16. Banka S, Chervinsky E, Newman WG, et al. Further delineation of the phenotype of severe congenital neutropenia type 4 due to mutations in G6PC3. Eur J Hum Genet 2011;19:18-22.

17. Banka S, Newman WG. A clinical and molecular review of ubiquitous glucose-6-phosphatase deficiency caused by G6PC3 mutations. Orphanet J Rare Dis 2013;8:84.

18. Vilboux T, Lev A, Malicdan MC, et al. A congenital neutrophil defect syndrome associated with mutations in VPS45. N Engl J Med 2013;369:54-65. 